\title{
Aplicaciones de la teoría implosión/ explosión: relación entre la Región Metropolitana de Santiago de Chile y los territorios productivos regionales
}

José Francisco Vergara-Perucich. Universidad Católica del Norte, Antofagasta, Chile.

RESUMEN | Desde la teoría de la implosión/explosión propuesta por Neil Brenner, tomando como base las interpretaciones de la urbanización planetaria desarrollada por Henri Lefebvre, se propone generar una nueva conceptualización teórica del problema de la urbanización, incorporando a la matriz de análisis los ciclos productivos económicos que ocurren en la no-ciudad cuyo capital resultante se acumula en la ciudad. Para evaluar la pertinencia de esta teoría en Chile, se analiza la relación entre volumen de producción mineral y forestal por regiones y las ventas de estas actividades económicas según región. El resultado es que la mayor parte de las ventas ocurren en Santiago, cuando gran parte de la producción ocurre en otras regiones. Se determina que es pertinente aplicar la teoría urbana de la implosión/explosión para el caso chileno y se proponen líneas investigativas a partir de la interpretación de las cartografías aquí presentadas.

PALABRAS CLAVE | urbanización, relación campo-ciudad, descentralización.

ABSTRACT | Based on Neil Brenner's Implosion/Explosion theory, this article suggests evidence for a new theoretical conceptualization of the urbanization phenomena. The methodology considers the inclusion of the economic cycles of production that occur outside the city to the matrix analysis. The importance of this theory relies on the significant dissociation between capital accumulation spaces and productive territories. By analyzing the relationship between the Mining and Forestry production with sales associated to these economic activities, the article tests the pertinence of the application of the urban theory in the Chilean case. Furthermore, the research findings demonstrate that most of the sales occur in Santiago, whereas most of the production occurs in other regions. Therefore, the Implosions/Explosions urban theory results as pertinent for understanding some urbanization processes in Chile, and consequently, the article proposes research prospects based on the cartographies exposed in this article.

KEYWORDS | urbanization, countryside-city relationship, decentralization. 


\section{Introducción}

En Historia de la acumulación capitalista de Chile, Gabriel Salazar (2003) explica que a partir de la empresa precapitalista instalada por Pedro de Valdivia en Chile en el siglo XVI, se desarrolló una burguesía mercantil colonial que concentraría el capital obtenido por las actividades comerciales de la colonia para su transacción internacional, consolidando un centro estratégico en el eje Santiago (centro protofinanciero) y Valparaíso (puerto de embarque de mercancías). Salazar expone que esta lógica condujo a un enfrentamiento comercial entre los mercaderes y los productores. Mientras los productores se encontraban asentados en los territorios productivos, labrando la tierra y generando productos para la exportación (en el siglo xvir fueron principalmente agrícolas y ganaderos), fueron los mercaderes los que desarrollaron la comercialización de esos bienes en el mercado colonial. Sin trabajar la tierra ni producir sobre una base material, el mercader, era

una especie de Caín, que fue expoliando poco a poco a su hermano Abel, hasta postrarlo en permanente agonía. El conflicto entre la función mercantil-financiera y la función propiamente productiva implicó que, para desarrollarse acumulativamente, la empresa valdiviana tuvo que desdoblarse y volverse contra sí misma. Donde el ciclo acumulativo (atrapado monopólicamente por los grandes mercaderes) se constituyó en un enérgico y constante factor erosionante del ciclo productivo (Salazar, 2003, p. 48).

De esta forma, la lógica de un ciclo productor lejos de la ciudad y la acumulación de capital en la ciudad, en el caso chileno, presenta una tendencia que se remonta a los primeros años de colonización y que se mantiene hasta la actualidad. Si bien evidencia al respecto existe y es de pública circulación, la interpretación de este fenómeno desde recientes avances en materia de teoría urbana podría presentar interesantes aproximaciones a una crítica a la explotación capitalista del territorio chileno que integre los discursos disciplinares críticos del modelo neoliberal bajo un mismo marco interpretativo.

El presente artículo toma la teoría urbana de la implosión/explosión, desarrollada por Neil Brenner y su equipo del Urban Theory Lab de Harvard, como marco teórico para elaborar un conjunto cartográfico que permita visualizar las diferencias que existen entre la explotación de los territorios productivos regionales en Chile y la comercialización de dichos productos en Santiago, demostrando así que el fenómeno colonial observado por Salazar se mantiene, con nuevos alcances y complejidades. La teoría de la implosión/explosión plantea que la conceptualización urbana ha tendido a considerar únicamente las problemáticas que ocurren dentro de los límites de lo que se entiende por ciudad, excluyendo del análisis aquello que está fuera de los límites o la no-ciudad. Para Brenner y su equipo, incorporar la no-ciudad a la matriz interpretativa de la teoría urbana contemporánea es vital para comprender cabalmente cómo los procesos capitalistas se alimentan de la producción del espacio. Los alcances de esta teoría son múltiples, tanto en la construcción de una crítica del capitalismo como para el desarrollo de alternativas. 
En este artículo se reúnen y mapean los datos sobre el volumen y dimensiones de la explotación de territorios productivos para los sectores económicos minero y forestal, para luego ponerlos en relación con las ventas de dichos sectores por región, demostrando así que mientras gran parte de los procesos productivos ocurren en regiones, las ventas se siguen concentrando en la capital. Si bien esto no pareciera ser un dato muy revelador, dado que es una especie de verdad asumida, la existencia de cartografías sobre tal realidad es escasa. En estas circunstancias, el artículo busca contribuir con argumentos espacializados a la discusión sobre la descentralización del país, permitiendo con ello que disciplinas como el diseño urbano, la planificación y la arquitectura aporten a dicha discusión con sus propias herramientas metodológicas.

Mediante recursos disponibles en instituciones públicas, se han reunido datos económicos y administrativos de las actividades mineras y forestales. Se ha buscado mapear el volumen de producción según región, la superficie asignada para realizar explotación productiva por región, la cantidad de empresas por rubro inscritas en cada región y el monto de ventas por región. En el particular de esta información, se ha consultado en ministerios, asociaciones gremiales, el Instituto Nacional de Estadísticas y en el Servicio de Impuestos Internos. Luego se ha buscado mapear la localización de la dirección comercial de las principales empresas de cada sector según ranking de América Economía y se han consultado los datos financieros y comerciales de dichas empresas en la Superintendencia de Valores y Seguros. Se asume que para configurar una muestra aún más representativa de estas cartografías, se podría haber realizado una consulta a las patentes comerciales de cada municipalidad, junto con la Ley de Rentas Municipales, las acciones de responsabilidad social empresarial de cada empresa analizada y la Ley de Presupuesto anual. Sin embargo, el objetivo de este mapeo no es evaluar las políticas públicas sobre descentralización o a favor de la equidad, sino más bien comprobar cuál es la relación entre territorios productivos y territorios de comercialización para dos de las principales actividades económicas del país.

Los datos recopilados, dirigidos a evaluar la pertinencia de la teoría de implosión/ explosión propuesta por Neil Brenner para analizar los problemas de desigualdad territorial y urbanización en Chile, pueden ser además un aporte a otras investigaciones.

\section{Implosión de una centralidad/explosión en el territorio}

El fenómeno de la implosión/explosión, según Henri Lefebvre (1972 [1970]), corresponde a una metáfora de la física nuclear que utiliza para describir un proceso de fuerte concentración territorial de las personas, actividades, riqueza, bienes, objetos, instrumentos, medios y pensamientos que caracterizan una realidad urbana, el cual desencadena una explosión que proyecta una serie de fragmentos, como periferias, suburbios, hogares vacacionales, barrios satélites y otras consecuencias espaciales, que se insertan lejos de los paños urbanos tradicionales para generar nuevos sistemas de utilización del territorio. Para Lefebvre, el punto de implosión/explosión ocurre con la modernidad y se caracteriza, fundamentalmente, por la total subordinación del territorio a las necesidades de la vida urbana. Esta subordinación obedece a las lógicas del capital, donde la acumulación de riqueza se genera gracias a la explotación del territorio productivo, sea este agrario, minero, pesquero, forestal o con 
fines inmobiliarios, por mencionar algunos ejemplos propios del caso chileno. Con la noción de implosión/explosión como fenómeno histórico, Lefebvre se refiere principalmente al caso europeo y las consecuencias de la posguerra. Para el caso latinoamericano, por cierto, el momento de implosión/explosión se asociará en este artículo a la implementación del modelo neoliberal, caracterizado por la profundización de un modelo económico extractivista de materias primas, socialmente apropiado por una elite político-económica dominante y con metas políticas a corto plazo, que apunta al crecimiento anual medido por el producto interno bruto y el empleo.

Paralelamente a los desarrollos teóricos de Lefebvre, en 1969 Fernando Henrique Cardoso y Enzo Faletto desarrollaban la teoría de la dependencia; y en 1974, Immanuel Wallerstein escribía su ensayo sobre los modern world-system, que confluirían ante una nueva condición planetaria en que el mundo comenzaba a tejer un conjunto de sólidas redes de interacción económica, donde unos subyugaban a otros. Cardoso y Faletto (1969) plantearán la existencia de relaciones de poder desiguales en el territorio global, donde las naciones pobres del sur explotan sus recursos naturales para abastecer a las naciones ricas del norte, generando ciclos de dependencia reproducidos a partir de sanciones económicas, política exterior, fuerzas armadas y por las mismas relaciones de mercado, en una escala planetaria. Por su parte, la teoría del modern world-system de Wallerstein (1974) localiza un centro mundial en el noroeste de la Europa moderna (siglo xvi), que concentra la acumulación capitalista a nivel global y establece la existencia de un centro, de una semiperiferia y una periferia, reconociendo así la validez de la teoría de la dependencia. Tal como lo explican Fujita, Krugman y Venables (2001), en economía urbana el supuesto más aceptado proviene del modelo de Von Thûnen de 1826, que básicamente plantea la búsqueda de la optimización de localización en relación con la cercanía de un actor a los principales mercados, lo que propiciaría la consolidación de un centro urbano por sobre un territorio productivo. En concreto, la relación centro-periferia, mercado-producción, implosión-explosión no es nueva en el estudio de la territorialización de los fenómenos capitalistas. El foco, entonces, no está en la lógica centro-periferia, sino en los efectos puntuales generados por este modo de producción, dado que existe un cambio de escala y, con ello, de los involucrados en el proceso. La industria no es más un espacio confinado entre muros, sino que es planetaria. Siguiendo con la metáfora, si el planeta se ha convertido en un gigantesco capital productivo, donde existe un espacio de extracción y un espacio de comercialización y posterior acumulación de capital, ¿`cómo se diseña y territorializa este fenómeno?

En una conferencia celebrada en The Bartlett School of Planning en diciembre de 2013, Neil Brenner planteaba que la urbanización contemporánea requiere una nueva conceptualización teórica que no solo abarque los centros urbanos, sino, principalmente, los efectos que ella tiene en el espacio de zonas más alejadas de las ciudades. La demanda de teorización planteada por Brenner surge desde una pregunta inicial: ¿cuáles son los efectos que tiene la urbanización de ciudades en los territorios localizados entre estas ciudades? Plantea al respecto que existen diversos campos $\operatorname{ciegos}^{1}$ que

"Campo ciego" es una categoría de análisis desarrollada por Henri Lefebvre en el libro La revolución urbana, de 1970, en que se refiere a construcciones de límites sociales, culturales, económicos y políticos que impiden avanzar en la producción de conocimiento. Son barreras que determinan lo posible y lo imposible, lo realista y lo utópico, verdades asumidas contra verdades por descubrir. 
condicionan una mirada centralizadora del problema urbano e impiden una comprensión más integral del problema. El primer campo ciego es el fetichismo por los asentamientos urbanos, caracterizado por un deseo de entender el problema urbano a partir de la ciudad. Un segundo campo ciego es la categorización de lo rural y de lo urbano como unidades de análisis separadas o bien como un continuo de lo urbano hacia lo rural, la cual genera una definición algo rígida de los procesos territoriales que conforman las lógicas del capital. El tercer campo ciego es la categorización de los cambios demográficos basada en una distribución de personas en una ruralidad o en una urbanidad, la cual genera una relación binaria para un problema que tiene mucha mayor complejidad. Para mejorar el análisis del capitalismo planetario y sus efectos en el espacio, según Brenner, es fundamental conceptualizar lo no-urbano, esos suelos que han sido considerados en los análisis por oposición a la vida urbana, no como parte constitutiva de ella, y tampoco en su capacidad de condicionar la vida urbana. De esta forma, para Brenner, la lógica de los centros urbanos debe ser comprendida desde una urbanización extendida, de una ciudad de ocho billones de habitantes.

Cualquiera sea la composición demográfica, desde las densas redes urbanas de las llanuras del Ganges o Java a los terrenos baldíos de Siberia o la estepa del desierto del Gobi, los espacios de la no-ciudad han sido continuamente operacionalizados para sustentar los procesos de construcción urbana a lo largo de la historia mundial de un desarrollo capitalista desigual. Por lo tanto, estos espacios son estratégicamente centrales a los procesos de destrucción creativa que soportan la "urbanización del capital" como son los grandes y densos centros urbanos que han monopolizado largamente la atención de los urbanistas (Brenner, 2016, pp. 123-124). ${ }^{2}$

Para Andy Merrifield (2014), el proceso de consolidación de la ciudad global, interpretada como una máquina de crecimiento económico, terminará por abrumar aceleradamente la vida urbana. En ella la sociedad queda subyugada a los procesos acumulativos, mientras los espacios representan la inequidad del poder y la riqueza que engrandecen a ciertos espacios globales (ciudades de la acumulación como Londres, París y Nueva York) y enrarecen la vida en las zonas marginales, víctimas del mercado mundial. Merrifield define este proceso como neo-haussmannización por la existencia de un centro desarrollado y una periferia empobrecida a costa del centro. La problematización de esta relación centro-periferia tiene una importante componente inicial basado en la comprensión de cómo este fenómeno opera a diversas escalas. En nuestro caso, si bien a escala planetaria existe un proceso mediante el cual el cobre chileno se comercializa en Londres principalmente, también existe un proceso en que el cobre producido en Chile se extrae en un territorio y se comercializa en Santiago, como también existe una tercera aproximación escalar en que

2 Traducción propia. El texto original de Brenner es el siguiente: "Whatever their demographic composition, from the dense town networks of the Ganges Plain or Java to the barren wastelands of Siberia or the Gobi desert steppe, the spaces of the non-city have been continuously operationalised in support of the city-building processes throughout the global history of capitalist uneven development. Such spaces are, therefore, as strategically central to the processes of creative destruction that underpin the 'urbanisation of capital' as are the large, dense urban centres that have long monopolised the attention of urbanists" (Brenner, 2016, pp. 123-124) 
un trabajador minero extrae el mineral mientras otro trabajador ingeniero diseña su instalación en el mercado.

$\mathrm{Al}$ respecto, lo que viene desarrollando Neil Brenner con el Urban Theory Lab de Harvard ofrece importantes aportes metodológicos para comprender de qué manera una alta centralización urbana afecta a las unidades territoriales productivas y la calidad de vida que se desarrolla en dichas unidades. Dado que estos procesos teóricos se encuentran en desarrollo, la construcción de cartografías de análisis resulta vital para aportar a una conceptualización con datos empíricos y posibilitar así el desarrollo de una crítica. En el caso chileno, por lo pronto, resulta relevante comprender, dentro de la escala nacional, cómo se configuran los ciclos de implosión/explosión, que - según el levantamiento historiográfico de Salazar- llevan siglos de implementación.

Valenzuela, Penaglia y Basaure (2015) establecen que este proceso de centralización territorial tiene una raíz política histórica, caracterizada por una concentración del producto interno bruto en la Región Metropolitana que no se explica por su aumento de población. A juicio de los investigadores, ello obedece más bien a un diseño centralista hoy obsoleto, que ha decantado en la organización de movimientos regionalistas alimentados por el descontento nacido de las desigualdades representadas en inequidades territoriales. "Tal desequilibrio los lleva a sentirse desfavorecidos por el sistema impositivo y por las consecuencias negativas de las actividades económicas, como inflación y contaminación. Se trata, así, de una crisis de expectativas entre el potencial económico de una región y su desarrollo real" (Valenzuela et al., 2015, p. 234). La crítica se representa al revisar los montos de inversión pública efectiva por regiones, como se indica en la tabla 1 .

TABLA I | Porcentaje de inversión pública efectiva total por regiones, 2014

\begin{tabular}{|l|c|c|}
\hline \multicolumn{1}{|c|}{ REGIÓN } & MILLONES DE PESOS & \% INVERSIÓN POR REGIÓN \\
\hline Arica y Parinacota & 105.000 & 2,3 \\
\hline Tarapacá & 110.000 & 2,4 \\
\hline Antofagasta & 170.000 & 3,7 \\
\hline Atacama & 120.000 & 2,6 \\
\hline Coquimbo & 310.000 & 6,8 \\
\hline Valparaíso & 350.000 & 7,7 \\
\hline Metropolitana & 850.000 & 18,7 \\
\hline O'Higgins & 302.000 & 6,6 \\
\hline Maule & 395.000 & 8,7 \\
\hline Biobío & 610.000 & 13,4 \\
\hline Araucanía & 335.000 & 7,4 \\
\hline Los Ríos & 207.000 & 4,6 \\
\hline Los Lagos & 402.000 & 8,8 \\
\hline Aysén & 140.000 & 3,1 \\
\hline Magallanes & 140.000 & 3,1 \\
\hline
\end{tabular}

FUENTE ELABORACIÓN PROPIA CON BASE EN DATOS DEL MINISTERIO DE DESARROLLO SOCIAL, SISTEMA NACIONAL DE INVERSIONES 
El desequilibrio que hacen patente las cifras señaladas acarrea problemas interregionales, como lo es el fenómeno de la conmutación, que para Patricio Aroca y Miguel Atienza (2008) corresponde a un proceso de movilidad constante de trabajadores entre regiones por razones laborales; esto es, tienen su fuente laboral en una región, pero viven en otra. Para Aroca y Atienza, tal fenómeno tiene una incidencia negativa en la calidad de vida de las regiones que resultan atractivas únicamente para trabajar, como es el caso de Antofagasta, dado que los ingresos generados en la región se utilizan en otra. Los autores puntualizan que "de los más de dieciséis mil trabadores que conmutan a la Región de Antofagasta, casi el 75 por ciento tiene su residencia a más de 800 kilómetros (...). Puede asumirse, por tanto, que el supuesto de separación entre el lugar de trabajo y el de consumo es válido en el caso de Antofagasta" (Aroca \& Atienza, 2008, p. 112). El estudio expone datos de un período en el cual la economía minera se encontraba en auge. Pero aun en períodos de crisis minera, como a finales de 2016, la conmutación de trabajadores a la Región de Antofagasta era de 50.000 trabajadores (según ha precisado Atienza recientemente), lo que representa un $25 \%$ de la fuerza laboral de la región. A partir de este análisis, se podrían desarrollar nuevas escalas de análisis para el problema del transporte público, el cual, retomando el análisis de Brenner, estando confinado a unidades territoriales urbanas, pierde eficacia a nivel interregional. También se puede instalar una discusión sobre la segregación residencial, donde los salarios se pagan en regiones, pero la inversión y consumo de los mismos se ejecutan en específicas centralidades urbanas, donde la interacción entre los trabajadores conmutados y los territorios sociales se encuentra reducida.

En esta línea argumental y buscando la representación geográfica de estos fenómenos en tanto Chile como una gran comuna de la ciudad planetaria, corresponde avanzar hacia producir una teoría apropiada para el estudio de esta nueva escala. Estos procesos abarcan no solo los límites urbanos definidos políticamente, sino también las operaciones territoriales destinadas a la producción. Resulta relevante rastrear el origen de las materias primas, por ejemplo, y el lugar donde se comercializan y se acumula el capital resultante de estas operaciones. Para el particular del caso Chile, mapear estas relaciones permitirá alimentar discusiones sobre descentralización, legislación en materia de patentes comerciales y la distribución de la riqueza, entre otros asuntos de pleno interés nacional. Si bien existe conciencia de la alta centralización del país, el desarrollo de una cartografía de análisis para esta condición permitirá avanzar hacia una conciencia espacial del problema, dimensionando apropiadamente la magnitud de la centralización y los efectos concretos de la implosión/explosión.

\section{Cartografías en fase de explosión}

Para elaborar las cartografías se han escogido los rubros minero y forestal, a fin de desarrollar mapas que expongan, principalmente, la relación entre la explotación del territorio productivo regional y las ventas anuales por sector económico. En primer orden, la elección de estos sectores productivos obedece a la búsqueda de rubros exportadores, para lo cual resultaban apropiados la minería, la pesca y los rubros 
agropecuario y forestal. Entre estos sectores, los que tenían mayor incidencia en la composición del producto interno bruto eran minería, con un $9,18 \%$, forestal, con $2,6 \%$, y agropecuario, con $1,7 \%$. También ha incidido en la decisión la selección de actividades productivas que ocupen importantes superficies territoriales y que generen transformaciones significativas en el suelo sobre el cual operan. De estos tres sectores se han escogido los de mayor incidencia en el PIB.

La conformación de esta muestra considera el ańo 2014, dado que es el último período comercial disponible para análisis en las bases de datos del Servicio de Impuestos Internos al momento de confeccionar los mapas. Existiendo una importante fragmentación de la información pública disponible, se han utilizado datos oficiales provistos por diversos servicios públicos, entre los que se cuentan el Servicio Nacional de Geología y Minería (Sernageomin), la Superintendencia de Valores y Seguros (svs), el Servicio de Impuestos Internos (sir), el Instituto Forestal (Infor) y el Instituto Nacional de Estadísticas (INE). Todos los mapas van apoyados por tablas para que se consulten los datos con mayor precisión.

\section{Primera cartografía: minería}

En la Región Metropolitana existen 1.102 concesiones mineras, inferiores en cantidad a las 12.671 en Antofagasta, o en relación a las 13.051 en Atacama (tabla 2). Sin embargo, comparativamente, las ventas por actividades económicas vinculadas a la minería a nivel regional indican una realidad contradictoria. Por ejemplo, la suma de las ventas por concepto de minería en las regiones de Tarapacá, Antofagasta y Atacama da uf 282.549.271, mientras en la Región Metropolitana el valor transado corresponde a UF 994.394.208, casi cuatro veces más que la suma de las regiones categorizadas por sus actividades mineras. Mientras en regiones se desarrolla una alta cantidad de actividades productivas mineras que aportan sustantivamente al crecimiento económico de Chile, la evidencia muestra que las ganancias por las ventas resultantes de estos procesos se concentran en Santiago. Esta complejidad se explica al ser Chile un estado unitario, no federado, razón por la cual, desde el punto de vista legal, no se infringe ninguna ley al extraer en regiones y comercializar en Santiago, disociando la localización territorio explotado con el domicilio comercial de la empresa explotadora.

Así, también existe una indiscutible diferencia en cuanto a las proporciones del territorio que se explota en las tres regiones del norte grande y la Región Metropolitana. Mientras la primera, segunda y tercera región suman un total de 9.657.100 hectáreas de superficie concesionada, en la Región Metropolitana únicamente se suscriben 307.300 hectáreas de explotación para la minería (tabla 2). 
TABLA 2 | Actividad minera en Chile por región, año 2014

\begin{tabular}{|c|c|c|c|c|}
\hline REGIÓN & $\begin{array}{l}\text { PRODUCCIÓN DE } \\
\text { MINERAL } \\
\text { (TMF / TONELADA } \\
\text { MÉTRICA FINA) }\end{array}$ & $\begin{array}{l}\text { NÚMERO DE } \\
\text { CONCESIONES } \\
\text { MINERAS }\end{array}$ & $\begin{array}{c}\text { HECTÁREAS } \\
\text { VIGENTES DE } \\
\text { EXPLOTACIÓN }\end{array}$ & VENTAS (UF) \\
\hline Arica y Parinacota & 1.003 .328 & 2.843 & 858.900 & 761.699 \\
\hline Tarapacá & 684.860 & 6.875 & 2.152 .600 & 102.814 .602 \\
\hline Antofagasta & 13.490 .466 & 12.671 & 3.977 .500 & 148.943 .846 \\
\hline Atacama & 11.731 .500 & 13.051 & 3.527 .000 & 30.790 .823 \\
\hline Coquimbo & 3.040 .701 & 5.274 & 1.585 .400 & 50.620 .869 \\
\hline Valparaíso & 2.380 .793 & 1.401 & 436.900 & 6.760 .013 \\
\hline Metropolitana & 6.278 .332 & 1.102 & 307.300 & 994.394 .208 \\
\hline O'Higgins & 1.853 .413 & 1.559 & 425.600 & 7.478 .011 \\
\hline Maule & 1.713 .960 & 1.961 & 540.000 & 2.453 .763 \\
\hline Biobío & 414.973 & 2.497 & 649.500 & 4.890 .185 \\
\hline Araucanía & 0 & 757 & 178.400 & 2.444 .881 \\
\hline Los Ríos & 0 & 469 & 117.900 & 881.015 \\
\hline Los Lagos & 0 & 1.133 & 336.800 & 1.508 .336 \\
\hline Aysén & 41.082 & 1.203 & 300.700 & 6.688 .327 \\
\hline Magallanes & 5.165 .666 & 1.237 & 339.700 & 19.050 .071 \\
\hline Total & 47.799 .074 & 54.033 & 15.734 .200 & 1.380 .480 .649 \\
\hline
\end{tabular}

Pasando desde una escala nacional al particular de las empresas que operan en esta lógica de disociar actividades extractivas con las ventas de dichas actividades, el $42,5 \%$ de las quince principales empresas mineras nacionales concentra sus oficinas centrales en Santiago, particularmente en un polígono conformado por calle Amunátegui hacia el poniente, Manquehue hacia el oriente, y con el eje AlamedaProvidencia-Apoquindo como conector. En este polígono se transan uf 322.439.897 (tabla 3). Por lo demás, muchas de estas empresas son internacionales, por lo que también se establece que la desterritorialización de estos procesos comerciales no ocurre únicamente dentro de los límites nacionales, sino que también participan en ello empresas australianas, británicas, suizas, sudafricanas, canadienses y estadounidenses, conectándose así con la centralidad planetaria de la potencial ciudad global.

Como se puede revisar en la cartografía a continuación (figura 1), la concentración de ventas por actividades mineras describe un adentro, un interior, entendido como el área de la Región Metropolitana, donde se ubican los domicilios comerciales de las empresas que concentran las ventas por la explotación del afuera, entendido como las áreas productivas lejanas a estos núcleos de concentración. Esto comprobaría la existencia de un proceso de implosión en cuanto a concentrar actividades de venta en un punto particular de la geografía urbana, y una explosión, expresada a través de la ubicación de medios productivos en áreas lejanas del centro de acumulación. 
TABLA 3 Domicilio comercial y ganancias por exportaciones de las 15 principales empresas mineras de Chile

\begin{tabular}{|c|c|c|c|c|c|}
\hline & EMPRESA & COMUNA & $\begin{array}{c}\text { TRANSACCIONES } \\
\text { EN UF }\end{array}$ & $\begin{array}{c}\text { \% DEL } \\
\text { TOTAL } \\
\text { NACIONAL }\end{array}$ & CONTROLADO POR: \\
\hline 1 & Codelco Chile & Santiago & 240.311 .704 & 14,77 & Estado de Chile \\
\hline 2 & Minera Escondida Ltda. & Las Condes & 142.527 .456 & 8,76 & BHP Billition (Australia / GB) \\
\hline 3 & Anglo American Sur s.A. & Providencia & 52.715 .634 & 3,24 & Anglo American \\
\hline 4 & Minera Los Pelambres & Las Condes & 52.064 .824 & 3,20 & Amsa \\
\hline 5 & $\begin{array}{l}\text { Cía. Metalúrgico Alto } \\
\text { Norte s.A. }\end{array}$ & Antofagasta & 42.790 .777 & 2,63 & Glencore \\
\hline 6 & $\begin{array}{l}\text { Cía. Dońa Inés de } \\
\text { Collahuasi }\end{array}$ & Providencia & 40.838 .346 & 2,51 & $\begin{array}{l}\text { Anglo American y } \\
\text { Glencore }\end{array}$ \\
\hline 7 & Minera Centinela s.A. & Las Condes & 39.048 .618 & 2,40 & Amsa \\
\hline 8 & Minera Spence s.A. & Las Condes & 28.472 .951 & 1,75 & BHP Billition (Australia / GB) \\
\hline 9 & $\begin{array}{l}\text { Cía. Contractual Can- } \\
\text { delaria }\end{array}$ & Las Condes & 24.405 .386 & 1,50 & Lundin Mining \\
\hline 10 & $\begin{array}{l}\text { Sociedad Contractual } \\
\text { el Abra }\end{array}$ & Calama & 21.964 .848 & 1,35 & Freeport McMoRan \\
\hline 11 & Cía. del Pacífico s.A. & La Serena & 18.710 .796 & 1,15 & CAP \\
\hline 12 & SQM Salar S.A. & Las Condes & 15.294 .042 & 0,94 & Julio Ponce Lerou \\
\hline 13 & Mantos Copper s.A. & Providencia & 14.317 .827 & 0,88 & Audley Capital Advisors \\
\hline 14 & Minera Cerro Colorado & Las Condes & 12.690 .801 & 0,78 & BHP Billition (Australia / GB) \\
\hline 15 & SQM Industrial s.A. & Las Condes & 12.528 .098 & 0,77 & Julio Ponce Lerou \\
\hline & \multicolumn{3}{|l|}{ Total } & $46,63 \%$ & \\
\hline
\end{tabular}

FIGURA I | Mapas de producción minera y de ventas según región, año 2014

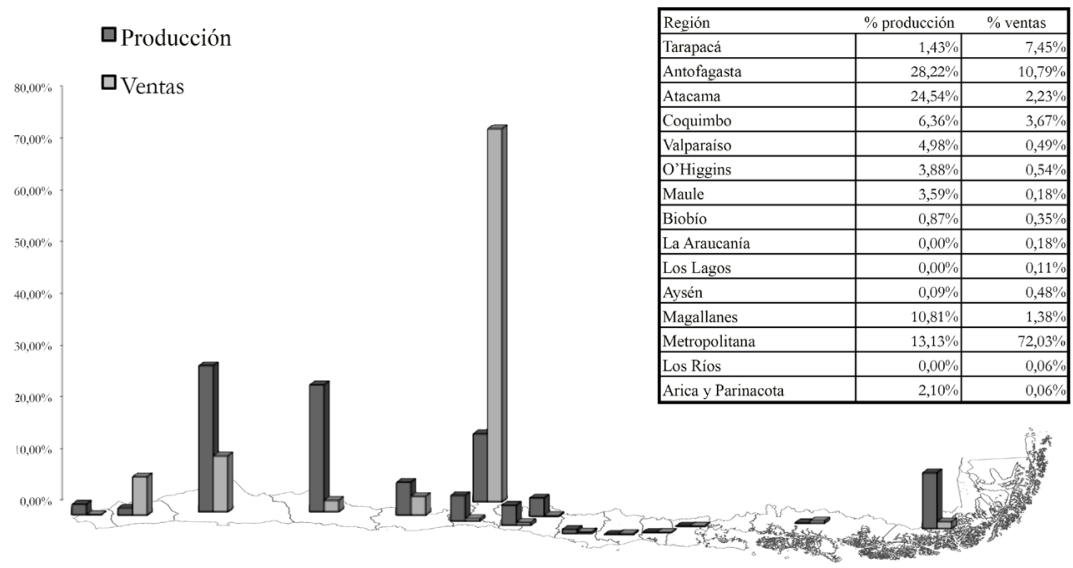

FUENTE ELABORACIÓN PROPIA 


\section{Segunda cartografía: forestal}

Tomando como base la producción forestal anual del año 2014, la región del Biobío es por lejos la que mayor aporta a este sector económico nacional. Con $4.383 .500 \mathrm{~m}^{3}$ de madera aserrada en 2014, esta región contribuye con el 55\% de la explotación de madera del país. Para lograrlo, han tenido que plantar bosque productivo en 923.506 hectáreas, lo que produce importantes alteraciones al ecosistema, al reemplazar bosque nativo por bosque productivo (Aylwin, Yáńez \& Sánchez, 2013). Como se indica en la tabla 4, en las regiones del Maule, del Biobío y de la Araucanía se desarrollan $6.998 .600 \mathrm{~m}^{3}$ de madera aserrada, alcanzando un $88 \%$ de la producción del país, mientras que en la Región Metropolitana es cero. No obstante, siguiendo el patrón de desterritorialización de las ventas, en esas tres regiones únicamente se vende un $18 \%$ del total por actividades forestales nacionales, mientras en Santiago se concentra un $80 \%$ de las ventas. Si bien tanto las actividades mineras como las forestales pueden afectar los modos de vida de las comunidades aledańas a los territorios productivos, en el caso del desarrollo económico de tipo forestal el enfrentamiento entre las comunidades locales y las iniciativas empresariales ha llegado a violencia efectiva, en conflictos de larga data y compleja solución. De esta forma, además de desterritorializar las ganancias de sus zonas productivas, los conflictos con las comunidades se desarrollan lejos de la centralidad nacional. Ello da cuenta de un proceso de resistencia entre las comunidades contra los administradores de las faenas, y no necesariamente contra los dueńos de los medios de producción y de la tierra en disputa, quienes residen lejos de estos puntos de conflicto. Se confirma con ello que la tesis originaria de la fundación de la economía chilena planteada por Salazar sigue vigente.

TABLA 4 | Actividad forestal en Chile por región, año 2014

\begin{tabular}{|l|r|r|r|r|}
\hline \multicolumn{1}{|c|}{ REGIÓN } & $\begin{array}{c}\text { PRODUCCIÓN } \\
\text { DE MADERA } \\
\text { ASERRADA (M }\end{array}$ & $\begin{array}{c}\text { NÚMERO DE } \\
\text { EMPRESAS } \\
\text { SILVÍCOLAS }\end{array}$ & $\begin{array}{c}\text { HECTÁREAS } \\
\text { DE BOSQUE } \\
\text { PRODUCTIVO }\end{array}$ & VENTAS (UF) \\
\hline Arica y Parinacota & 0 & 2 & 0 & 0 \\
\hline Tarapacá & 0 & 8 & 0 & 0 \\
\hline Antofagasta & 0 & 5 & 0 & 0 \\
\hline Atacama & 0 & 7 & 84.152 & 0 \\
\hline Coquimbo & 29.400 & 279 & 47.642 & 2.927 .049 \\
\hline Valparaíso & 0 & 644 & 6.361 & 590.671 .658 \\
\hline Metropolitana & 178.400 & 290 & 126.999 & 3.481 .499 \\
\hline O’Higgins & 1.714 .800 & 922 & 460.271 & 10.600 .182 \\
\hline Maule & 4.383 .500 & 2.050 & 923.506 & 112.128 .084 \\
\hline Biobío & 900.300 & 1.026 & 494.390 & 11.130 .711 \\
\hline Araucanía & 607.700 & 627 & 185.915 & 6.421 .197 \\
\hline Los Ríos & & & & 0 \\
\hline
\end{tabular}

(continúa) 
(continuación)

\begin{tabular}{|l|c|c|c|c|}
\hline \multicolumn{1}{|c|}{ REGIÓN } & $\begin{array}{c}\text { PRODUCCIÓN } \\
\text { DE MADERA } \\
\text { ASERRADA (M }\end{array}$ & $\begin{array}{c}\text { NÚMERO DE } \\
\text { EMPRESAS } \\
\text { SILVÍCOLAS }\end{array}$ & $\begin{array}{c}\text { HECTÁREAS } \\
\text { DE BOSQUE } \\
\text { PRODUCTIVO }\end{array}$ & VENTAS (UF) \\
\hline Los Lagos & 135.800 & 569 & 75.839 & 3.290 .022 \\
\hline Aysén & 9.900 & 120 & 42.515 & 232.604 \\
\hline Magallanes & 38.200 & 56 & 0 & 69.733 \\
\hline Total & 7.998 .000 & 6.653 & 2.447 .590 & 740.952 .740 \\
\hline
\end{tabular}

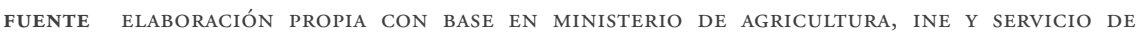
IMPUESTOS INTERNOS

TABLA 5 | Domicilio comercial y ganancias de diez grandes empresas forestales de Chile

\begin{tabular}{|c|c|c|c|c|c|}
\hline & EMPRESA & $\begin{array}{c}\text { COMUNA } \\
\text { DOMICILIO DE } \\
\text { LA EMPRESA }\end{array}$ & VENTAS UF & $\begin{array}{c}\text { \% DEL } \\
\text { TOTAL } \\
\text { FORESTAL }\end{array}$ & $\begin{array}{c}\text { CONTROLADO } \\
\text { POR: }\end{array}$ \\
\hline 1 & Empresa CMPC & Santiago & 127.736 .492 & 17,24 & Grupo Matte \\
\hline 2 & Arauco & Las Condes & 132.127 .474 & 17,83 & Grupo Angelini \\
\hline 3 & смрс Tissue & Santiago & 47.604 .922 & 6,42 & Grupo Matte \\
\hline 4 & смPC Celulosa & Santiago & 46.077 .065 & 6,22 & Grupo Matte \\
\hline 5 & MASISA & Las Condes & 35.043 .118 & 4,73 & Grupo Nueva \\
\hline 6 & CMPC Papeles & Santiago & 28.582 .468 & 3,86 & Grupo Matte \\
\hline 7 & CMPC Productos de Papel & Santiago & 10.011 .953 & 1,35 & Grupo Matte \\
\hline 8 & Aserraderos Arauco & Las Condes & 18.829 .866 & 2,54 & Grupo Angelini \\
\hline 9 & СмPC Forestal Mininco & Santiago & 20.840 .474 & 2,81 & Grupo Matte \\
\hline 10 & Forestal CELCO & Santiago & 26.707 .956 & 3,60 & Grupo Angelini \\
\hline & \multicolumn{3}{|c|}{ Total \% } & 66,61 & \\
\hline
\end{tabular}

FUENTE ELABORACIÓN PROPIA CON BASE EN DATOS DE LA SUPERINTENDENCIA DE VALORES Y SEGUROS Y DEL BANCO CENTRAL DE CHILE

En relación con las empresas que presentan las mayores ganancias por actividades forestales, se observa un patrón de concentración no solo de dichas ganancias, sino también de los grupos controladores. Mientras las ganancias de las diez principales empresas alcanza un 66,61\% del total producido a nivel nacional, nueve de ellas son controladas o por el grupo Matte o por el grupo Angelini. Además de existir una implosión territorial, también aparece una concentración económica en dos conglomerados (tabla 5). Al igual que con la minería, estas empresas también se ubican en un polígono urbano conformado por calle Amunátegui hacia el poniente y Manquehue hacia el oriente.

En definitiva, uf 493.561 .788 por ventas forestales se declaran en las comunas de Providencia y Las Condes, donde no existe ni una sola hectárea de bosque productivo. A diferencia del caso de la minería, las principales empresas controladoras de esta actividad económica son chilenas. A pesar de no constituir una ilegalidad ni mucho menos, tanto el caso minero como el forestal sugieren una nueva magnitud de análisis para los procesos de urbanización. En el caso forestal, la disociación de 
los territorios productivos y de los conflictos sociales que surgen a partir de estos procesos (figura 2), junto con el anterior antecedente, constituyen argumentos a favor de aplicar la teoría de la implosión/explosión en el caso chileno y, consiguientemente, un aporte desde Chile a una necesidad global de conceptualizar la ciudad a partir de una totalidad planetaria.

FIGURA 2 | Mapa de producción forestal y ventas según región, año 2014

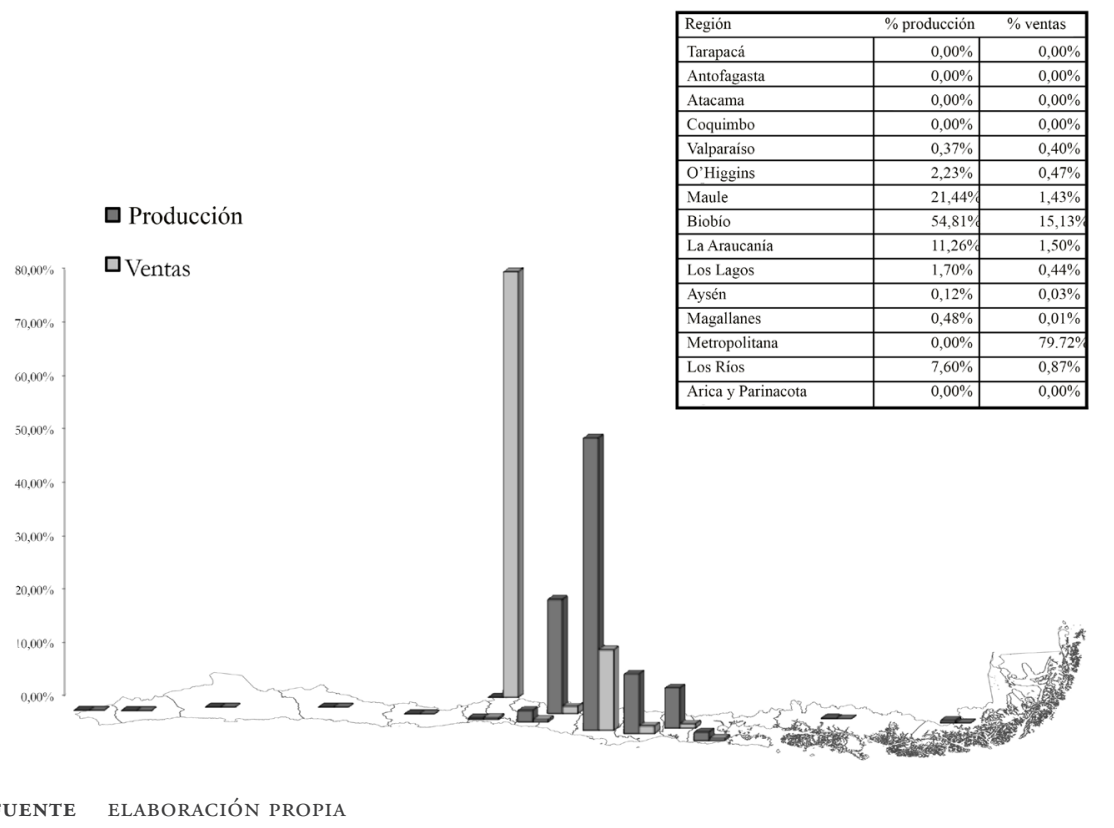

Conclusiones y explosiones:

la importancia de la totalidad teórica transdisciplinar

Tal y como lo plantea Neil Brenner, la teoría urbana contemporánea no puede ignorar que gran parte de la producción capitalista del espacio en la ciudad depende de una explotación capitalista del espacio en la no ciudad, por lo que la escala de análisis de la ciudad debe comenzar a incorporar las redes económicas que alimentan el proceso de desarrollo de la ciudad. Asumiendo la existencia de una condición de urbanización planetaria, el estudio de la ciudad comienza donde se originan las materias primas para crear procesos de acumulación de capital en ciertas centralidades urbanas planetarias. Los datos aquí presentados aportan cartográficamente al análisis de dicha realidad; no obstante, su profundización y especificación necesita nuevas derivadas. Esto implica desarrollar investigaciones científicas sobre cómo el capital generado por los procesos productivos en regiones se transforma en productos urbanos, y evaluar si además de concentrar las ventas de estas materias primas existe una consecuente acumulación de capital en centralidades intermedias de circuitos globales, como sería el caso de Santiago, a medio camino entre el punto 
de extracción y el destino de comercialización. En un análisis secuencial, desde este punto metodológico se deberá avanzar en comprender de qué forma estos productos urbanos derivados, desarrollados con el capital resultante de los procesos productivos, se reproducen. Es decir, tomar la teoría urbana sobre la implosión/explosión y utilizarla para ver de qué manera las materias primas se convierten en capital urbano y de qué forma el capital urbano, para reproducirse, degrada el territorio de la no-ciudad.

Al mapear los datos oficiales, desde una aproximación general al problema de la diferencia entre territorios productivos y territorios acumulativos, se establece que el fenómeno de la implosión/explosión es real y que, en el caso chileno, se requiere avanzar desde la descripción geográfica de este fenómeno al análisis urbano-planetario de prácticas productivas que decantan en transformaciones espaciales. En otras palabras, desde la teoría urbana se debe pasar a revelar cómo estos procesos de explotación y acumulación condicionan los modos de vida de las personas.

Existen diversas investigaciones en curso sobre los procesos de explotación y acumulación en particular, aunque, dada la especificidad de cada caso presentado, no logran reunirse en una teoría capaz de totalizar una crítica al modelo capitalista cuya robustez se funde en una teoría general soportada por diversos casos que la informen. La teoría de la implosión/explosión pareciera dar una pauta para producir, desde el caso chileno, una crítica integral al proceso de urbanización planetaria. Así, las investigaciones sobre empleo (Rehner, Rodriguez \& Murray, 2018), descentralización (Valenzuela et al., 2015), conmutación (Aroca \& Atienza, 2008), gentrificación (López Meza \& Gasic, 2014), desposesión (Guerra \& Skewes, 2010), segregación (Ruiz-Tagle, 2016), fragmentación (Jirón \& Mansilla, 2014), participación (Elizalde, De la Maza \& Córdova, 2013) y enclaves (Arias, Atienza \& Cademártori, 2014), por mencionar algunos tópicos usados en recientes estudios urbanos, podrían confluir bajo un mismo enfoque teórico capaz de reunirlas y generar un discurso político basado en información científica sobre problemáticas propias de la urbanización. Esta búsqueda, si bien expone una importante ambición intelectual y se enfrentaría al complejo desafío de organizar diversos cuerpos académicos, podría ayudar a que el problema de la urbanización retome una posición de relevancia en la agenda política, dejando de lado la fragmentación que la caracteriza en la actualidad.

A nivel de la ciudad, la concentración de las ventas por actividades mineras y forestales se ve reflejada en un entorno construido cuya calidad espacial se ve exageradamente mejorada en relación con las áreas del territorio productivo nacional.

Si bien los datos aquí provistos no son suficientes para determinar una directa relación entre el proceso de concentración geográfica del capital empresarial forestal y minero con la calidad del espacio urbano, la evidencia otorga viabilidad a una investigación al respecto. Así, se podría establecer que los procesos de segregación espacial no son urbanos, en tanto contenidos en ciudades, sino que son nacionales y tienen una escala de país, o bien planetaria, como insinuaría la teoría de Brenner. Avanzando más allá de los aportes cartográficos y hacia la articulación propositiva con la teoría de la implosión/explosión, se podría establecer una hipótesis sobre la existencia de una directa relación entre la distancia desde los núcleos 
de concentración de las ventas y los ciclos de vida de las ciudades. Así, mientras un territorio productivo está condenado a depender del agotamiento de sus recursos útiles para el capital, el lugar donde se concentran las ventas dependerá únicamente de mantener modos de producción activos en la no-ciudad, sin importar cuáles sean ni dónde se ubiquen. De esta manera, un centro financiero en el contexto actual está destinado al éxito, mientras que un territorio únicamente productivo estará condenado a ver su fin. Esta hipótesis resulta preocupante no solo para el caso de las regiones de Antofagasta o del Biobío, sino para regiones donde urge el desarrollo de diversificación productiva.

FIGURA 3 Polígono donde se concentra el 42,5\% de las ventas de empresas de minería y el $66,61 \%$ de las ventas de empresas forestales

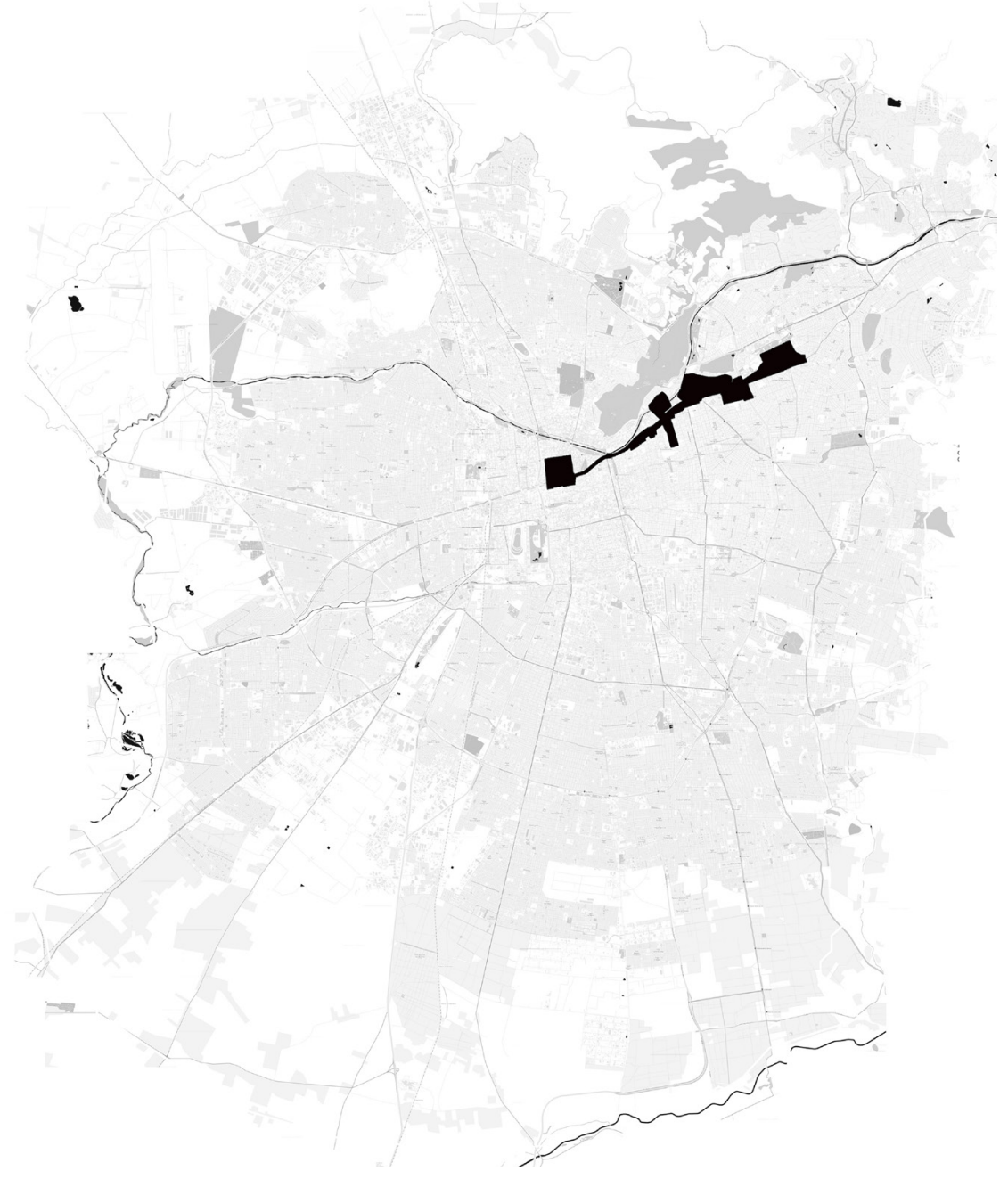

FUENTE ELABORACIÓN PROPIA 
Este fenómeno de concentración capitalista como consecuencia de explotación productiva del territorio no es exclusivo del caso chileno y, desde la perspectiva de la producción del espacio, ha sido largamente teorizado desde mediados de los años setenta, tanto en Chile como en el resto del mundo. En 1989, Henri Lefebvre escribiría un ensayo llamado "Cuando la ciudad se pierda en una metamorfosis planetaria", ${ }^{3}$ donde exponía cómo el aburguesamiento de los centros urbanos los había convertido no solo en lugares de consumo, sino también en objetos de consumo, expulsando a los productores hacia la periferia, convirtiéndolos en verdaderos turistas de la ciudad a la cual se encuentran políticamente vinculados. Para Lefebvre, esta situación era el resultado de una intensificación de un proceso histórico de concentración en una centralidad, que daba cuenta de un modelo urbano que comenzaba a cubrir todo el territorio global, amenazando con una homogeneización del espacio y la total eliminación de la diversidad, para dejar crecer los patrones de diferenciación, es decir, que cada espacio sea mono-funcional. Los espacios -plantea Lefebvre- serán fragmentados de acuerdo con su rentabilidad para ser vendidos y esto ayudará a establecer nuevas jerarquías en relación con las clases sociales, en espacialidades por clases planetarias. Esta inquietante interpretación apunta a producir un objeto virtual crítico sobre el futuro de lo que se podría entender como el avance a transformar el planeta en una gran urbanidad. Al respecto, en 1989, Carlos De Mattos ya advertía que

[d] esde el punto de vista territorial, seguramente el resultado más importante de los cambios reseñados es que en estos procesos productivos protagonizados por grandes empresas y por grupos económicos en expansión, las estrategias empresariales que realmente establecen la dirección central de la dinámica de acumulación de cada sistema se orientan hacia una progresiva transectorialización y transregionalización del capital. Este tipo de estrategia está conduciendo en forma inexorable a una paulatina y progresiva superación de aquella etapa del desarrollo capitalista en la que los propietarios del capital tendían a identificarse con un determinado sector (comercial, agrícola, industrial) o con una determinada colectividad humana (urbana o rural) (p. 77).

Para De Mattos, la mayor complejidad ante la implementación del modelo económico imperante hasta hoy, posiblemente afectaría a aquellas regiones y territorios que al momento de iniciar este nuevo proceso ya presentaran ciertos atrasos y desigualdades, las cuales se verían acrecentadas por las lógicas del capital. Así, plantea como un futuro posible que la acumulación se desarrolle lejos de estos focos productivos, en centros que tengan una preexistencia en materia de acumulación de capital.

Para finalizar, la producción de estas cartografías a partir de la problemática desarrollada por la teoría de la implosión/explosión expone la viabilidad e importancia de aplicar esta teoría en la interpretación del futuro de las áreas productivas chilenas y prevenir su eventual colapso. Al menos en el cono sur ya existen investigaciones sobre diversas aproximaciones al problema de la relación adentro y afuera, entre 
la ciudad y la no-ciudad, por lo que el solo hecho de ponerlo bajo el lente de la teoría de Brenner, podría proveer de nuevas estrategias para avanzar en resolver un problema que, en Chile, se inició en 1541.

\section{Referencias bibliográficas}

Arboleda, M. (2015). Spaces of extraction, metropolitan explosions: planetary urbanization and the commodity boom in Latin America. International Journal of Urban and Regional Research, 40(1), 96-112. https://doi.org/10.1111/1468-2427.12290.

Arias, M., Atienza, M. \& Cademártori, J. (2014). Large mining enterprises and regional development in Chile: between the enclave and cluster. Journal of Economic Geography, 14(1), 73-95. https://doi.org/10.1093/jeg/lbt007

Aroca, P. \& Atienza, M. (2008). La conmutación regional en Chile y su impacto en la Región de Antofagasta. EURE (Santiago), 34(102), 97-120. http://dx.doi.org/10.4067/ S0250-71612008000200006

Aylwin, J., Yáńez, N. \& Sánchez, R. (2013). Pueblo mapuche y recursos forestales en Chile: devastación y conservación en un contexto de globalización económica. Documento de trabajo. Santiago de Chile: Observatorio Ciudadano / Iwgia (Grupo de Trabajo Internacional para Asuntos Indígenas). https:/www.iwgia.org/images/ publications//0625_Pueblo_mapuche_y_recursos_forestales_en_chile.pdf

Brenner, N. (2013, 12 de diciembre). Urban theory without an outside. Centenary Lecture Series, en The Bartlett School of Planning, University College London. Londres, Reino Unido. Publicado en N. Brenner (ed.), Implosions/Explosions: Towards a Study of Planetary Urbanization (pp. 14-35). Berlín: Jovis.

Brenner, N. (2016). The hinterland urbanised? Architectural Design, 86(4), 118-127. https:// doi.org/10.1002/ad.2077

Cardoso, F. H. \& Faletto, E. (1969). Desarrollo y dependencia en América Latina. México, D.F.: Siglo XXI.

Daher, A. (2003). Regiones-commodities: crisis y contagio en Chile. EURE (Santiago), 29(86), 89-108. http://dx.doi.org/10.4067/S0250-71612003008600005

De Mattos, C. (1989). Reestructuración social, grupos económicos y desterritorialización del capital. El caso de los países del cono sur. EURE (Santiago), 16 (47), 61-90. http:// eure.cl/index.php/eure/article/view/1050

De Mattos, C. (2015). Revolución urbana. Estado, mercado y capital en America Latina. Santiago de Chile: Ril Editores.

Elizalde, A., De la Maza, G. \& Córdova, M. G. (2013). Sociedad civil y democracia en América Latina: desafíos de participación y representación. Polis. Revista Latinoamericana, 12(36), 7-12. http://dx.doi.org/10.4067/S0718-65682013000300001.

Guerra, M. D. \& Skewes, J.(2010). Acumulación por desposesión y respuestas locales en el remodelaje de los paisajes estuariales del sur de Chile. Chungará: Revista de Antropología Chilena, 42(2), 451-463. http://dx.doi.org/10.4067/S0717-73562010000200008

Harvey, D. (2007). A brief history of neoliberalism. Nueva York: Oxford University Press. 
Jirón, P. \& Mansilla, P. (2014). Las consecuencias del urbanismo fragmentador en la vida cotidiana de habitantes de la ciudad de Santiago de Chile. EURE (Santiago), 40(121), 5-28. http://dx.doi.org/10.4067/S0250-71612014000300001

Lefebvre, H. (1972 [1970]) La revolución urbana. Madrid: Alianza Editorial.

Lefebvre, H. (1989). Quand la ville se perd dans une métamorphose planétaire. Le Monde Diplomatique, mayo 1989, pp. 16-17. https://www.monde-diplomatique.fr/1989/05/ LEFEBVRE/41710

Lefebvre, H. (2003 [1970]) The urban revolution. Minneapolis, MN: University of Minnesota Press.

López, E., Meza, D. \& Gasic, I. (2014). Neoliberalismo, regulación ad-hoc de suelo y gentrificación: el historial de la renovación urbana del sector Santa Isabel, Santiago. Revista de Geografia Norte Grande, (58), 161-177. http://dx.doi.org/10.4067/S071834022014000200009

Merrifield, A. (2014). The new urban question. Londres: Pluto Press.

Montoya Arango, V. (2007). The map of the invisible: Silences and the grammar of power in cartography. Universitas Humanistica, (63), 155-180. http://www.scielo.org.co/scielo. php?script=sci_arttext $\&$ pid $=$ S0120-48072007000100009

Rehner, J., Rodríguez, S., \& Murray, W. E. (2018). Ciudades en auge en Chile: rol de la actividad exportadora en la dinámica del empleo urbano. EURE (Santiago), 44(131), 151-171.

Ruiz-Tagle, J. (2016). La persistencia de la segregación y la desigualdad en barrios socialmente diversos: un estudio de caso en La Florida, Santiago. EURE (Santiago), 42(125), 81108. http://dx.doi.org/10.4067/S0250-71612016000100004

Salazar, G. (2003). Historia de la acumulación capitalista de Chile-Santiago: LOM.

Valenzuela, E., Penaglia, F., \& Basaure, L. (2015). Acciones colectivas territoriales en Chile, 2011 2013: de lo ambiental-reivindicativo al autonomismo regionalista. EURE (Santiago), 42(125), 225-250. http://dx.doi.org/10.4067/S0250-71612016000100010

Wallerstein, I. M. (2005). Análisis de sistemas-mundo. Una introducción. México / Buenos Aires / Madrid: Siglo xxi. Versión PDF en http://www.multiversidadreal.edu.mx/wpcontent/uploads/2015/09/Wallerstein-Immanuel.pdf 


\section{Anexo}

FIGURA AI | Mapa síntesis de relación entre producción y ventas de actividades mineras
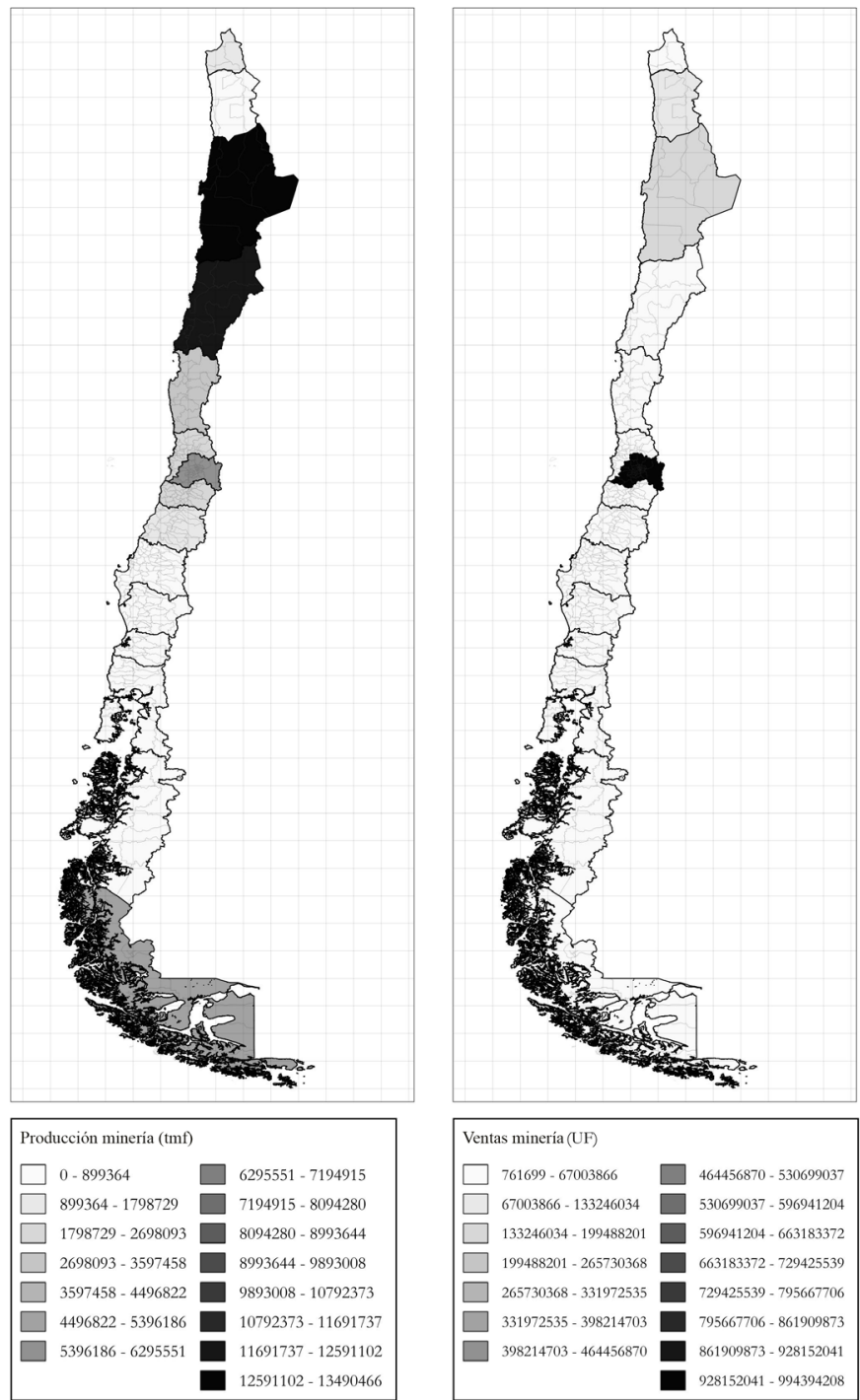

FUENTE ELABORACIÓN PROPIA 
FIGURA A2 | Mapa síntesis de relación entre producción y ventas de actividades forestales
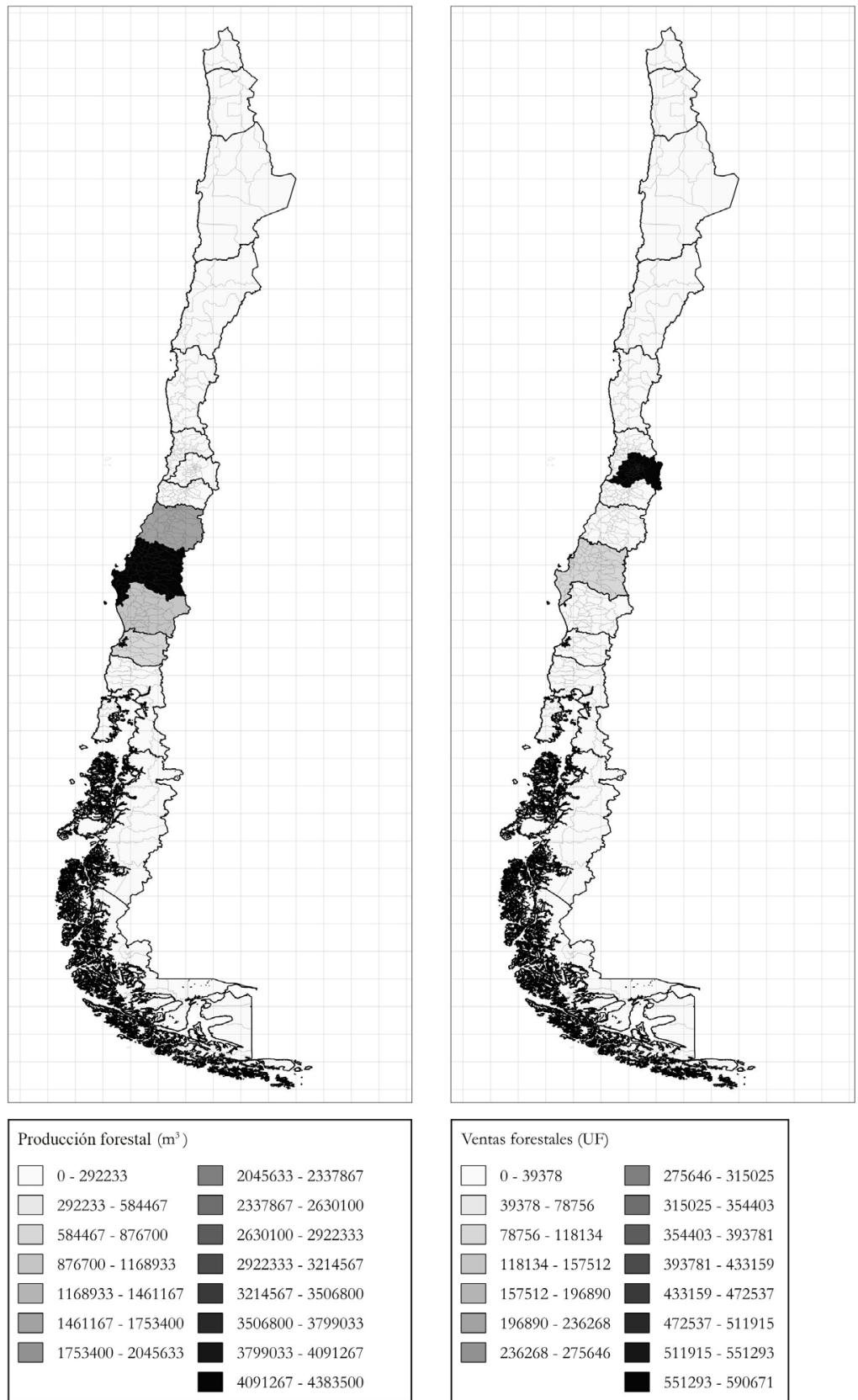

FUENTE ELABORACIÓN PROPIA 